Max-Planck-Institut für Kohlenforschung

This is the author's version of the work. It is posted here by permission of the AAAS for personal use, not for redistribution.

The definitive version was published in Science, 2018, 359, 1501-1505, DOI: 10.1126/science.aaq0445.

https://science.sciencemag.org/content/359/6383/1501

\title{
Activation of olefins via asymmetric Brønsted acid catalysis
}

\author{
Nobuya Tsuji ${ }^{1}$, Jennifer L. Kennemur ${ }^{1}$, Thomas Buyck ${ }^{1}$, Sunggi Lee ${ }^{1}$, Sébastien Prévost ${ }^{1}$, Philip S. J. \\ Kaib $^{1}$, Dmytro Bykov ${ }^{2}$, Christophe Farès ${ }^{1}$ and Benjamin List ${ }^{1 *}$ \\ ${ }^{1}$ Max-Planck-Institut für Kohlenforschung, Kaiser-Wilhelm-Platz 1, 45470 Mülheim an der Ruhr, Germany. \\ ${ }^{2}$ National Center for Computational Sciences, Oak Ridge National Laboratory, Oak Ridge, TN 37831. \\ ${ }^{*}$ Correspondence to: list@kofo.mpg.de
}

\begin{abstract}
The activation of olefins for asymmetric chemical synthesis traditionally relies on transition metal catalysts. In contrast, biological enzymes with Brønsted acidic sites of appropriate strength can protonate olefins and thereby generate carbocations that ultimately react to form natural products. Although chemists have recently designed chiral Brønsted acid catalysts to activate imines and carbonyl compounds, mimicking these enzymes to protonate simple olefins that then engage in asymmetric catalytic reactions has remained a significant synthetic challenge. Here we show that a new class of confined and strong chiral Brønsted acids enables the catalytic asymmetric intramolecular hydroalkoxylation of unbiased olefins. The methodology enables rapid access to biologically active 1,1-disubstituted tetrahydrofurans, including (-)-Boivinianin B.
\end{abstract}

One Sentence Summary: A confined and strong chiral Brønsted acid catalyzes the asymmetric intramolecular hydroalkoxylation of unbiased alkenes.

Main Text: Alkenes are a fundamental and ubiquitous class of organic compounds, often obtained in a single step from crude oil. Among the diverse synthetic applications of olefins, hydrofunctionalization reactions are prized due to their conceptual simplicity, perfect atom economy, and the increased value of the resulting products. $(1,2)$ Although such transformations have been extensively studied for decades using either Brønsted acid- or transition metalcatalysts, enantioselective variations remain challenging. For example, in contrast to catalytic asymmetric hydroaminations (3-5) or hydrofunctionalizations of dienes and allenes $(6,7)$, which are relatively well-developed, the enantioselective hydroalkoxylation of electronically neutral olefins is extremely rare, despite its great potential value. While asymmetric photocatalysis can provide anti-Markovnikov adducts in moderate enantioselectivities (8-10), asymmetric Markovnikov hydroalkoxylations are mainly studied using chiral metal catalysts. However a highly enantioselective variant with sterically and electronically unbiased olefins has so far not been described, despite major advances in asymmetric metal catalysis.(11-14) The lack of progress in this field is presumably due to socalled "hidden acid catalysis", referring to the in situ release of an achiral Brønsted acid that promotes the nonasymmetric background transformation. $(15,16)$ In contrast, enzymes can activate olefins by protonation and achieve excellent stereoselectivities.(17) Recently, an enzyme-catalyzed hydroalkoxylation involving an olefin protonation was reported, albeit with a limited substrate scope.(18)

Inspired by the extraordinary capacity of such enzymes to catalyze asymmetric functionalizations of simple olefins, we hypothesized that a well-designed chiral Brønsted acid could induce asymmetric chemical functionalizations of olefins with a more general scope. Such acids should, on the one hand, display very high acidity to enable olefin protonation, and on the other hand, provide a confined, enzyme-like microenvironment to favor the desired transformation with high selectivity, avoiding potential side reactions (Fig. 1A). Although chiral Brønsted acids are now widely-used catalytic motifs, enabling numerous asymmetric transformations, their use is still largely limited to the activation of imines and carbonyls. In stark contrast, unactivated alkenes have remained out of reach for current chiral Brønsted acid catalysts because of their weak basicity.(19-23) 
A
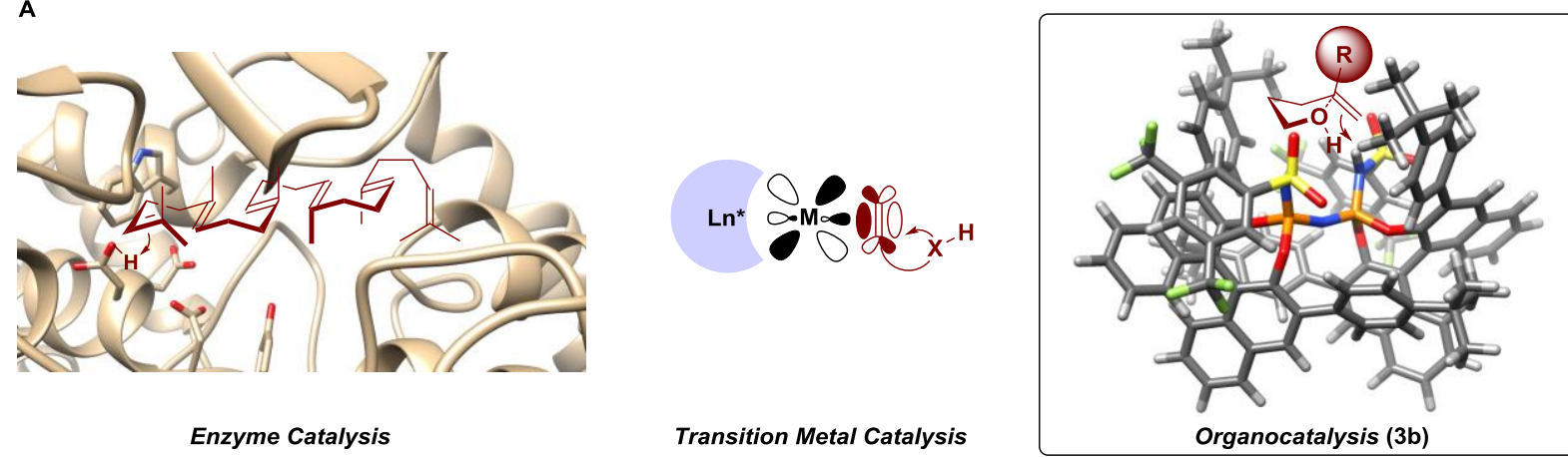

Enzyme Catalysis

Transition Metal Catalysis

Organocatalysis (3b)

B
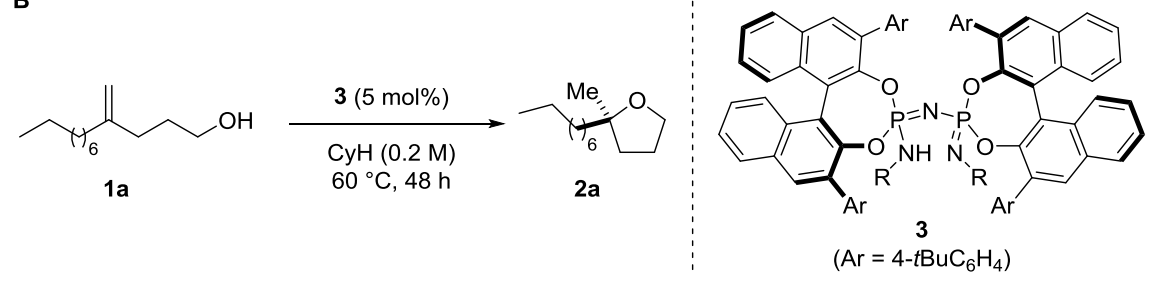

$\mathrm{R}=$

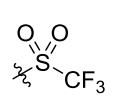

年"<smiles>[Y]S(=O)(=O)c1cc(C(F)(F)F)cc(C(F)(F)F)c1</smiles>

$3 a$

$86 \%$

3b

95\% $(91 \%)$

97.5:2.5 e.r

Fig. 1. (A) Three approaches for the activation of olefins. Shown from left to right are the enzyme squalene-hopene cyclase [PDB: 1SQC(17)], a general depiction of the well-defined activation of an olefin with a transition metal complex, and the posited binding mode of a strong and confined Brønsted acid organocatalyst. (B) Catalyst optimization. Yields were determined by ${ }^{1} \mathrm{H}$ NMR using mesitylene as an internal standard. Isolated yield in parentheses. $\mathrm{CyH}=$ cyclohexane.

We have previously introduced the chiral, confined imidodiphosphate (IDP) motif as a highly selective Brønsted acid catalyst that can convert small and unfunctionalized aliphatic substrates.(24) Recently, we have accomplished a substantial increase in the acidity of our IDPs by replacing the phosphoryl oxygen atoms with $N$-triflyl groups. The resulting imidodiphosphorimidate (IDPi) catalysts have proven to be highly acidic Lewis acid precatalysts of great utility in silylium-based Lewis acid organocatalysis.(25) We became interested in further exploring their utility in Brønsted acid catalysis,(26) envisioning that IDPi catalysts could potentially be used in the asymmetric activation of simple olefins.

Multisubstituted oxygen heterocycles are common motifs in natural products and pharmaceuticals such as polyketides.(27) The stereoselective synthesis of substituted tetrahydrofurans typically relies on the use of enantiomerically pure alcohols. We envisioned the direct asymmetric hydroalkoxylation of alkenols as a more straightforward and atom-economical approach. Indeed the electronically and sterically unbiased alkenol 1a was used as a model substrate to test the validity of our hypothesis (Fig 1B). Although most of the conventional Brønsted acid catalysts were inefficient (see supplementary materials), IDPi 3a turned out to be promising in terms of both reactivity and enantioselectivity. Towards identifying an optimal catalyst for our desired transformations, we realized an additional benefit of our IDPi design. Beyond the 3,3'-positions of the IDP catalysts, the two alkylsulfonyl substitutents of the IDPi motif offer an additional dimension for diversification and fine-tuning. Excellent yield and enantioselectivity were obtained using IDPi $\mathbf{3 b}$, bearing sterically more demanding arylsulfonyl groups at nitrogen, instead of triflyl groups. The most stable conformation of $3 \mathbf{b}$ was calculated based on the $\mathrm{x}$-ray structure of an $\mathrm{N}$-triflyl IDPi catalyst.(25) Because the larger substituent on the sulfonamide (R) is located in the confined environment, steric repulsions between $\mathrm{R}$ and the BINOL backbones presumably make the conformation more rigid and the cavity narrower in the transition states (see supplementary materials for a detailed discussion).

With an optimal catalyst and conditions in hand, we started investigating the scope and limitation of this hydroalkoxylation reaction (Fig. 2). In addition to different aliphatic substituents (2b, 2c), other functional groups, such as an ether (2d) and even an unprotected hydroxyl group (2e), were compatible under the reaction conditions. Sulfonamide-containing tetrahydrofuran $\mathbf{2 f}$, which possesses the core skeleton of the marketed pharmaceutical Mefruside,(28) was also obtained in excellent enantioselectivity. Substrates with tertiary $(\mathbf{2 g}, \mathbf{2 h})$ and even quaternary (2i) centers next to the alkene were compatible with this transformation. Dienes, which generate synthetically useful allylic ethers, were also readily converted (2j, 2k). To our delight, styrene derivatives also reacted to form the desired tetrahydrofurans in excellent yields and enantioselectivities. In addition to the phenyl- (2I) and naphthyl- (2m) groups, a variety of functional groups, such as aryl halides (2n, $2 \mathbf{0}$ and $\mathbf{S 2 a}$ ), an alkyl group (S2b), an isolated olefin (2p) and an ether (2q) were compatible. 


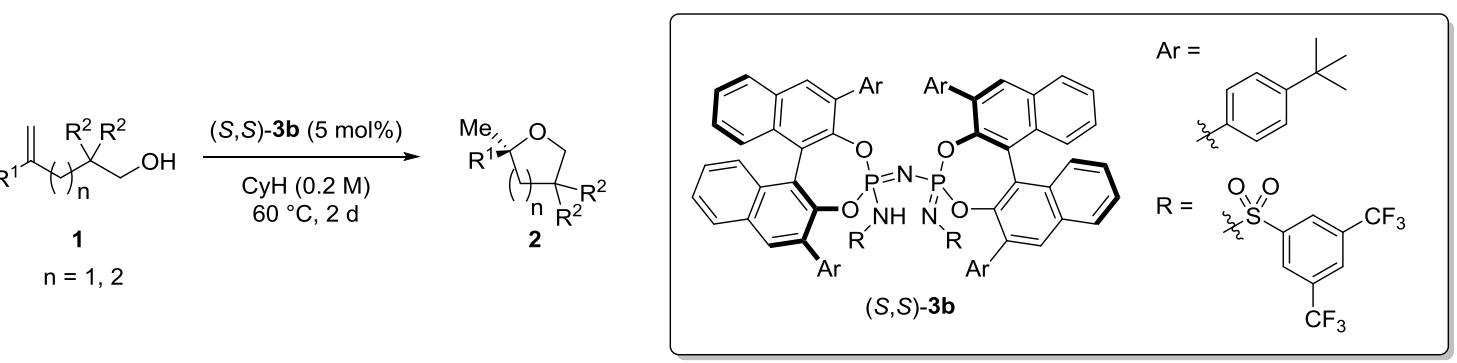

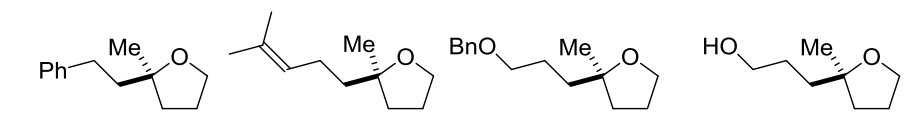

2b

89\%, 97.5:2.5 e.r.

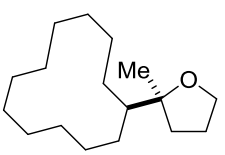

2h

$83 \%, 95.5: 4.5$ e.r.

(7d)
94\%, $95: 5$ e.r

(1 d) 2d

$94 \%, 97: 3$ e.r.

(1 d)

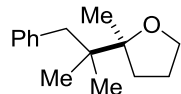

$2 \mathbf{i}$
$41 \%, 92: 8$ e.r

(0.5 M, $7 \mathrm{~d})$

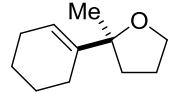

2j
2 e

$88 \%{ }^{*}, 94: 6$ e.r.

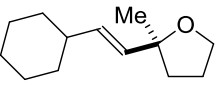

2k

$\left(10{ }^{\circ} \mathrm{C}, 7 \mathrm{~d}\right)$

$85 \%, 97.5: 2.5$ e.r. $\left(10^{\circ} \mathrm{C}, 7 \mathrm{~d}\right)$

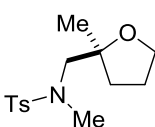

$2 f$

$66 \%, 97: 3$ e.r.

$\left(80^{\circ} \mathrm{C}, 7 \mathrm{~d}\right)$

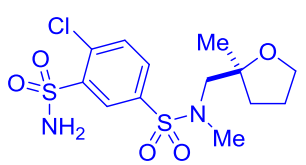

(S)-Mefruside

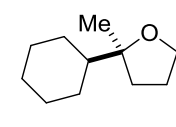

2g

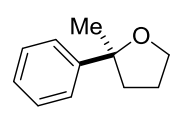

21

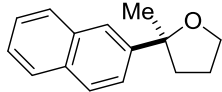

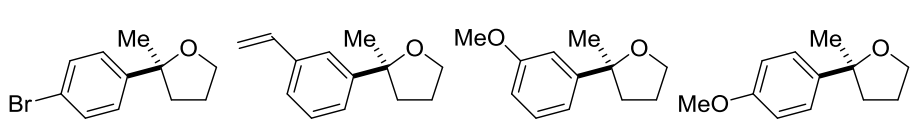

20

$94 \%, 98: 2$ e.r.

(4 d) 2p

$\mathbf{2 p}$
$76 \%, 98.5: 1.5$ e.r. $\quad 89 \%, 98: 2$ e.r.

$89 \%, 98: 2$ e.r.

$84 \%$

87\%, 2 $96.5: 3.5$ e.r.

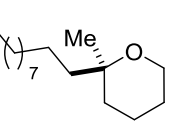

$2 \mathrm{t}$ $\begin{array}{cc}\text { 2r } & \text { 2s } \\ \text { 89\%, 94.5:5.5 e.r. } & 70 \%, 94: 6 \text { e.r }\end{array}$ (r.t., $1 \mathrm{~d}$ )

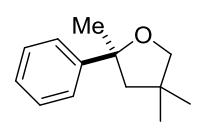

$70 \%, 92.5: 7.5$ e.r.

(7 d)

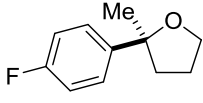

2n $83 \%, 97: 3$ e.r.

(5 d)

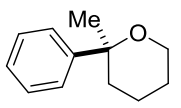

$2 u$ Fig. 2. Scope of the hydroalkoxylation. Isolated yields are presented. Enantiomeric ratios (e.r.) were determined either via HPLC or GC. "The reaction was performed in 1,2-dichloroethane-cyclohexane $(1: 1)$. $\mathrm{CyH}=$ cyclohexane, $\mathrm{Ts}=p$-toluenesulfonyl.

A methoxy-substituted styrene (1r) was also a good substrate. Although this electron-rich styrene might be considered activated for the cyclization, it could also have led to product racemization under the acidic conditions. Interestingly, a higher concentration and longer reaction time were required in the case of dimethyl-substituted styrene $1 \mathrm{~s}$ in order to achieve reasonable conversion, despite a possible Thorpe-Ingold effect. The bulky gemdimethyl group presumably hinders access of this substrate into the sterically demanding catalyst active site. We also envisioned the same strategy for the synthesis of substituted tetrahydropyrans. Like tetrahydrofurans, the tetrahydropyran skeleton is ubiquitous in natural products and pharmaceuticals, such that enantioselective syntheses of this motif are of great value. Indeed, 2,2-disubstituted tetrahydropyrans were obtained in good yields and slightly reduced enantioselectivities $(2 \mathbf{t}, \mathbf{2 u})$.

In addition to enantioselective hydroalkoxylations, diastereoselective versions may provide a valuable approach to natural products or pharmaceuticals with multiple stereogenic centers.(27) In contrast to 6-membered ring-forming cyclizations, which are often highly diastereoselective due to well-defined chair- or boat-like transition states, the diastereocontrolled synthesis of multisubstituted tetrahydrofurans is typically more difficult as the energetic difference between isomeric transition states is often smaller. We envisioned that a catalyst-controlled diastereoselective transformation that is independent of existing stereocenters would be powerful. In this vein, the two diastereoisomeric products $\mathbf{2} \mathbf{v}$ and $\mathbf{2} \mathbf{v}^{\prime}$ could be selectively obtained from alcohol $(S)-\mathbf{1} \mathbf{v}$, fully controlled by using either the $(S, S)$ or the $(R, R)$ enantiomer of the catalyst (Fig. 3A). Moreover, optically pure secondary alcohol $(R)$-1w selectively furnished 2,2,5-trisubstituted tetrahydrofurans $\mathbf{2} \mathbf{w}$ or $\mathbf{2} \mathbf{w}^{\prime}$ in excellent yields and diastereoselectivities, which were mainly controlled by the catalyst.

Encouraged by the wide scope of our method, a concise synthesis of (-)-Boivinianin B (29) was designed to demonstrate the utility, simplicity and efficiency of the developed transformation (Fig. 3B). Accordingly, alkenol 1x was subjected to the reaction conditions, using a lower loading of catalyst (1 mol\%), in a one-neck flask under air, to provide the corresponding tetrahydrofuran in excellent yield and enantioselectivity. The obtained product was oxidized without erosion of enantiopurity to furnish the corresponding lactone, which was confirmed to be (-)Boivinianin B by comparison to analytical data.(30) 
A

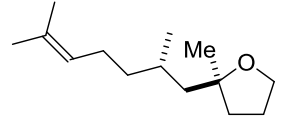

2v $81 \%,>20: 1$ d.r.

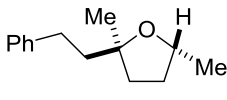

2w

$96 \%$ yield, $11: 1$ d.r.

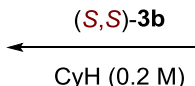

$60^{\circ} \mathrm{C}, 2 \mathrm{~d}$

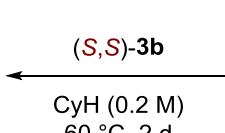

$60^{\circ} \mathrm{C}, 2 \mathrm{~d}$

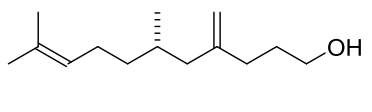

(S)-1v

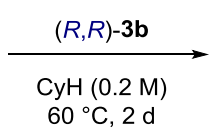

$60{ }^{\circ} \mathrm{C}, 2 \mathrm{~d}$<smiles>C=C(CCc1ccccc1)CCC(C)(C)O</smiles>

(R)-1w

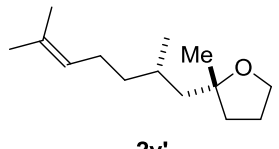

2v'

$75 \%$ yield, $>20: 1$ d.r.

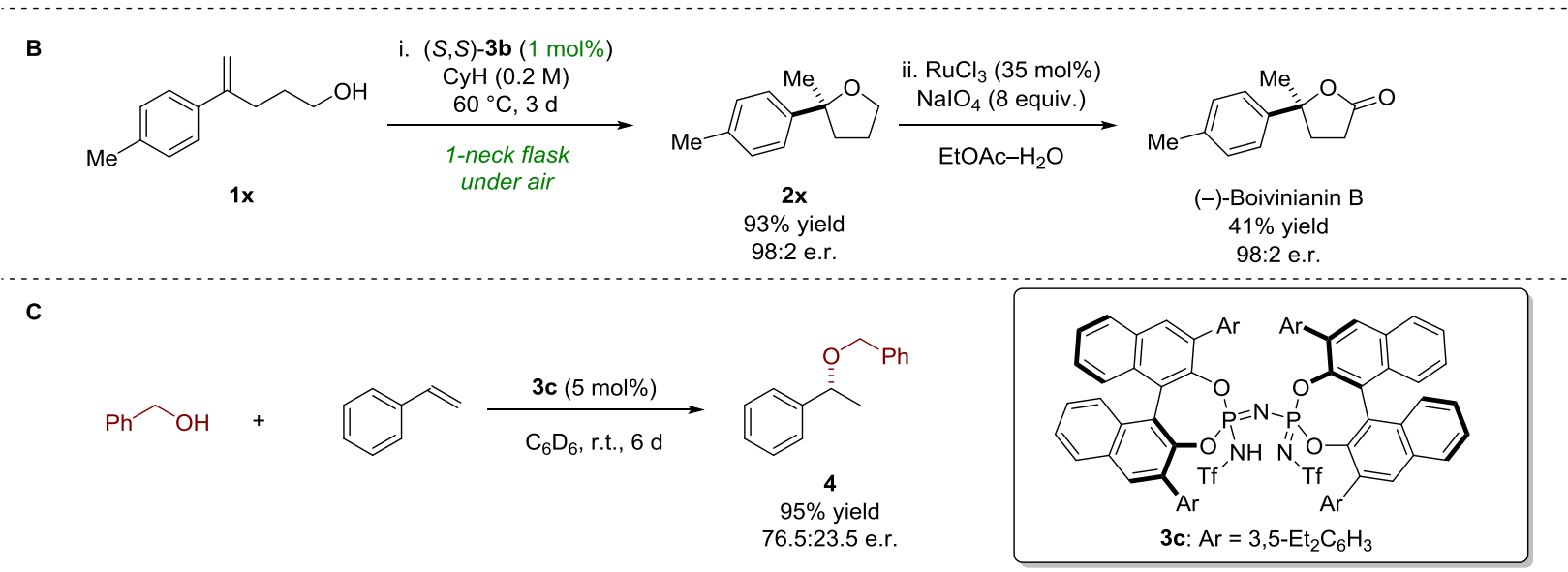

Fig. 3. Further application of the methodology. (A) Catalyst-controlled diastereoselective hydroalkoxylations. Diastereomeric ratios (d.r.) were determined by ${ }^{1} \mathrm{H}$-NMR and GC or HPLC. (B) Concise synthesis of $(-)$-Boivinianin $\mathrm{B}$. (C) A preliminary intermolecular hydroalkoxylation. CyH = cyclohexane.

To demonstrate the generality of this approach, we also investigated an intermolecular hydroalkoxylation, which is considered another major synthetic challenge with respect to both reactivity and enantioselectivity (Fig. 1C).(13) Gratifyingly IDPi $3 c$ catalyzed the reaction of styrene with benzyl alcohol to furnish product $\mathbf{4}$ in $95 \%$ yield and with very promising enantioselectivity (er $=76.5: 23.5)$. Further optimizations are currently ongoing.

Mechanistically, we initially envisioned the reaction to proceed via a stepwise protonation, cyclization pathway. However, a concerted mechanism in which protonation and $\mathrm{C}-\mathrm{O}$ bond formation occur more synchronously is also plausible $(18,31)$. Towards gaining more insight into the reaction mechanism and the origin of enantioselectivity, we performed density functional theory (DFT) studies (see supplementary materials for a description of the methods and a detailed discussion). The energies were obtained under B3LYP/def2-TZVP/D3(BJ)/CPCM level of theory. The lowest energy transition state TS1 suggests a concerted though asynchronous mechanism in which the reaction is triggered by the protonation of the olefin followed by C-O bond formation (Fig. 4A). The proposed transition state also describes the origin of enantioselectivities (Fig. 4B). Whereas the major enantiomer is generated via TS1 with 13.8 $\mathrm{kcal} / \mathrm{mol}$ of activation barrier, the minor enantiomer requires $15.4 \mathrm{kcal} / \mathrm{mol}$ to reach $\mathbf{T S} 1^{\prime}$. In addition, in TS1, the substituent of the substrate points towards the outside of the pocket due to the sterically demanding 3,3'-position of the BINOL backbones, which presumably allowed for the broad substrate scope.

To further evaluate the mechanism experimentally, a Hammett analysis with a series of styrene derivatives was performed (Fig $4 \mathrm{C}$ ). Plotting $\log \left(k_{\mathrm{x}} / \mathrm{k}_{\mathrm{H}}\right)$ against substituent parameter $\sigma^{+}$gave a linear correlation with a negative slope $(\rho=-2.08 \pm 0.04)$, which is consistent with the proposed carbocationic intermediate in the transition state. In order to gain further insights into the reaction mechanism, the corresponding alcohol $\mathbf{5}$ and olefin isomers $\mathbf{6}$ and $\mathbf{7}$ were subjected to the reaction conditions (Fig 4D). If the reaction indeed proceeds via a carbocation, either within an ion-pair or covalently bound to the catalyst, substrates 5-7 should provide essentially identical enantioselectivities. Whereas substrate $\mathbf{1 I}$ afforded the desired tetrahydrofuran $\mathbf{2} \mathbf{I}$ in excellent yield and enantioselectivity, $\mathbf{5}$ provided product $\mathbf{2 l}$ in only moderate yield and with poor enantioselectivity. Neither $\mathbf{6}$ nor $\mathbf{7}$ gave any of the desired products under these conditions or at higher temperature. These results are consistent with the proposed asynchronous concerted mechanism. 
A

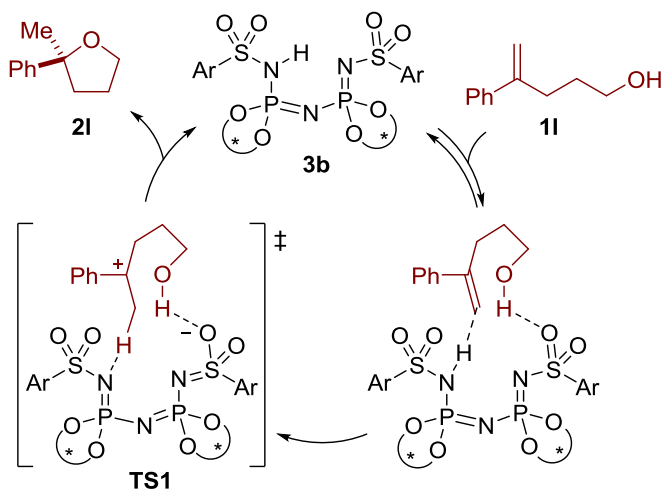

B

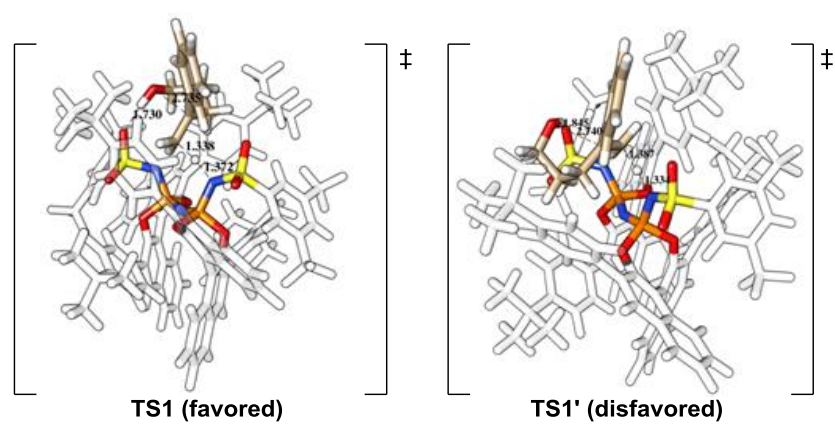

C<smiles>C=C(CCCO)c1ccccc1</smiles>

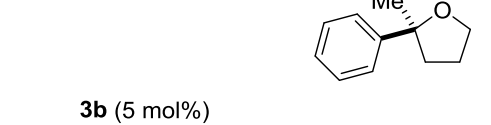<smiles>[X]c1ccc(C(=[CH+])CCCO)cc1</smiles>
Cyclohexane- $d_{12}$ $60{ }^{\circ} \mathrm{C}$ single pot $\mathrm{X}=\mathrm{OMe}, \mathrm{Me}, \mathrm{H}, \mathrm{F}, \mathrm{Cl}$, or $\mathrm{Br}$
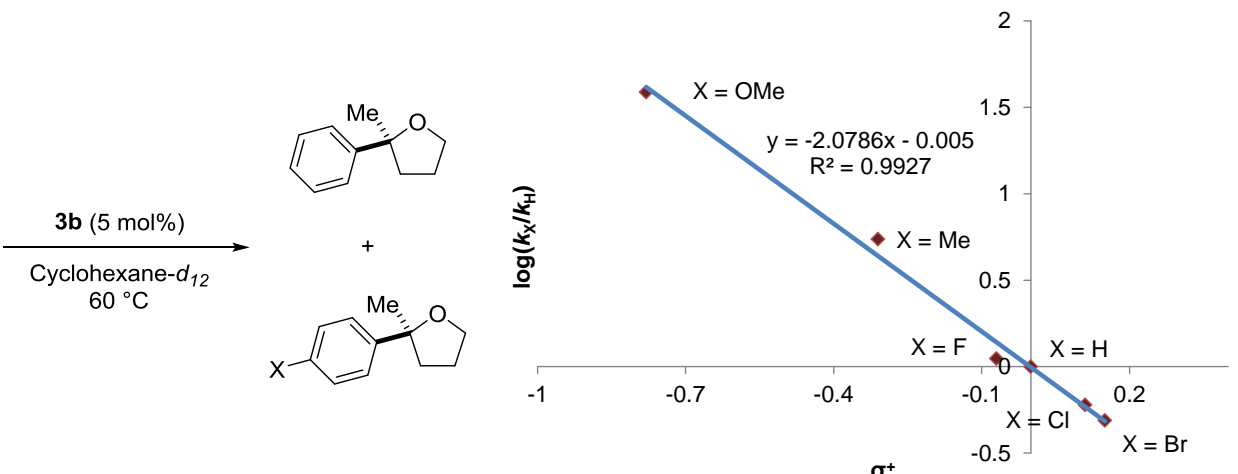

D substrate $\frac{3 \mathbf{b}(5 \mathrm{~mol} \%)}{\substack{\mathrm{CyH}(0.1 \mathrm{M}) \\ 60^{\circ} \mathrm{C}, 48 \mathrm{~h}}}$

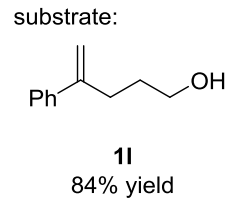

98.5:1.5 e.r.

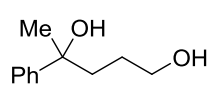

5

$45 \%$ yield 65:35 e.r.<smiles>C/C(=C\CCO)c1ccccc1</smiles>

\section{6}

$60^{\circ} \mathrm{C}$ : n.d. $80^{\circ} \mathrm{C}$ : n.d.

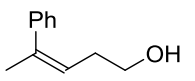

7

$60^{\circ} \mathrm{C}$ : n.d. $80^{\circ} \mathrm{C}$ : n.d.

n.d. $=$ not detected

Fig. 4. Mechanistic studies. (A) A plausible catalytic cycle. (B) DFT-calculated enantiodetermining transition states of the hydroalkoxylation of 1I. To increase visibility, the bulky substituents in the catalyst are whitened in this picture. All distances are in Angstroms. For more details, see the supplementary materials. (C) Intramolecular Hammett analysis $\left(\sigma^{+}\right)$is consistent with asynchronous concerted mechanism. For more details, see the supplementary materials. (D) Hydroalkoxylation with olefin isomers and etherification of the corresponding alcohol. $\mathrm{CyH}=\mathrm{cyclohexane}$.

The approach delineated here enables the asymmetric activation of electronically and sterically unbiased alkenes by highly confined and highly acidic organocatalysts. The methodology is operationally simple and a variety of alkenols could be converted in high yields and enantioselectivities. We believe that the presented approach bears great potential for various other hydrofunctionalizations and related reactions of olefins.

\section{References and Notes:}

1. M. Beller, J. Seayad, A. Tillack, H. Jiao, Angew. Chem. Int. Ed. 43, 3368-3398 (2004).

2. V. Rodriguez-Ruiz, R. Carlino, S. Bezzenine-Lafollée, R. Gil, D. Prim, E. Schulz, J. Hannedouche, Dalton Trans. 44, 1202912059 (2015)

3. E. Bernoud, C. Lepori, M. Mellah, E. Schulz, J. Hannedouche, Catal. Sci. Technol. 5, 2017-2037 (2015).

4. J.-S. Lin, Y. Peng, L. Huang, P. Zhang, B. Tan, X.-Y. Liu, Angew. Chem. Int. Ed. 54, 7847-7851 (2015).

5. L. Ackermann, A. Althammer, Synlett 7, 995-998 (2008).

6. N. D. Shapiro, V. Rauniyar, G. L. Hamilton, J. Wu, F. D. Toste, Nature 470, 245-250 (2011).

7. G. L. Hamilton, E. J. Kang, M. Mba, F. D. Toste, Science 317, 496-499 (2007).

8. D. S. Hamilton, D. A. Nicewicz, J. Am. Chem. Soc. 134, 18577-18580 (2012).

9. Y. Nishiyama, T. Wada, S. Asaoka, T. Mori, T. A. McCarty, N. D. Kraut, F. V. Bright, Y. Inoue, J. Am. Chem. Soc. 130, 75267527 (2008).

10. Z. Yang, H. Li, S. Li, M.-T. Zhang, S. Luo, Org. Chem. Front. 4, 1037-1041 (2017).

11. H. Murayama, K. Nagao, H. Ohmiya, M. Sawamura, Org. Lett. 17, 2039-2041 (2015).

12. J. Schlüter, M. Blazejak, F. Boeck, L. Hintermann, Angew. Chem. Int. Ed. 54, 4014-4017 (2015).

13. C. S. Sevov, J. F. Hartwig, J. Am. Chem. Soc. 135, 9303-9306 (2013).

14. H. Shigehisa, M. Hayashi, H. Ohkawa, T. Suzuki, H. Okayasu, M. Mukai, A. Yamazaki, R. Kawai, H. Kikuchi, Y. Satoh, A. Fukuyama, K. Hiroya, J. Am. Chem. Soc. 138, 10597-10604 (2016).

15. T. T. Dang, F. Boeck, L. Hintermann, J. Org. Chem. 76, 9353-9361 (2011).

16. D. C. Rosenfeld, S. Shekhar, A. Takemiya, M. Utsunomiya, J. F. Hartwig, Org. Lett. 8, 4179-4182 (2006). 
17. K. U. Wendt, K. Poralla, G. E.Schultz, Science 277, 1811-1815 (1997).

18. S.-S. Gao, M. Garcia-Borràs, J. S. Barber, Y. Hai, A. Duan, N. K. Garg, K. N. Houk, Y. Tang, J. Am. Chem. Soc. 139, 36393642 (2017).

19. M. Terada, Synthesis 12, 1929-1982 (2010).

20. G. Adair, S. Mukherjee, B. List, Aldrichimica Acta 41, 31-39 (2008).

21. D. Parmar, E. Sugiono, S. Raja, M. Rueping, Chem. Rev. 114, 9047-9153 (2014).

22. T. Akiyama, K. Mori, Chem. Rev. 115, 9277-9306 (2015).

23. A. Sakakura, M. Sakuma, K. Ishihara, Org. Lett. 13, 3130-3133 (2011).

24. I. Čorić, B. List, Nature 483, 315-319 (2012).

25. P. S. J. Kaib, L. Schreyer, S. Lee, R. Properzi, B. List, Angew. Chem. Int. Ed. 55, 13200-13203 (2016).

26. L. Liu, H. Kim, Y. Xie, C. Farès, P. S. J. Kaib, R. Goddard, B. List, J. Am. Chem. Soc. 139, 13656-13659 (2017).

27. A. Lorente, J. Lamariano-Merketegi, F. Albericio, M. Álvarez, Chem. Rev. 113, 4567-4610 (2013).

28. H. B. Allen, D. A. Lee, Curr. Med. Res. Opin. 1, 547-553 (1973).

29. D. A. Mulholland, K. McFarland, M. Randrianarivelojosia, Biochem. Syst. Ecol. 34, 365-369 (2006).

30. I. Corić, S. Müller, B. List, J. Am. Chem. Soc. 132, 17370-17373 (2010).

31. Y. Y. Khomutnyk, A. J. Argüelles, G. A. Winschel, Z. Sun, P. M. Zimmerman, P. Nagorny, J. Am. Chem. Soc. 138, 444-456 (2016).

Acknowledgements: We thank the GC department, the MS department, and the NMR department, especially Chris Hartding, of the MPI für Kohlenforschung for analytics. We thank Dr. Martin Klußmann for discussions about Hammett analysis and Dr. Chandra Kanta De for general discussions.

Funding: Generous support from the Max Planck Society, the Deutsche Forschungsgemeinschaft (Leibniz Award to B.L. and Cluster of Excellence RESOLV, EXC 1069), Swiss National Science Foundation (Fellowship to T.B.) and the European Research Council (Advanced Grant "C-H Acids for Organic Synthesis, CHAOS") is gratefully acknowledged. This research used resources of the Oak Ridge Leadership Computing Facility, which is a DOE Office of Science User Facility supported under Contract DE-AC05-00OR22725.

Author contributions: B.L. conceived and directed the project. N.T., S.L. and J.L.K. performed experiments and analysis. N.T. found the optimized catalyst and conditions. N.T. and S.L. jointly designed and prepared the catalyst. J.L.K. developed the intermolecular hydroalkoxylation. T.B., S.P. and P.S.J.K. contributed to initial studies. D.B. performed the computational studies. C.F. contributed to the NMR studies. N.T. and B.L. prepared the manuscript.

Competing interests: The authors declare no conflict of interest.

Data and materials availability: The supplementary materials are available online at http://www.sciencemag.org/

\section{Supplementary Materials:}

Materials and methods

Figures S1-S15

Table S1-S3

NMR spectra

HPLC traces

References (32-68) 